\title{
Experimental characterization of frequency-dependent squeezed light
}

\author{
Simon Chelkowski, Henning Vahlbruch, Boris Hage, Alexander Franzen, Nico Lastzka, \\ Karsten Danzmann, and Roman Schnabel \\ Institut für Atom- und Molekülphysik, Universität Hannover and Max-Planck-Institut für Gravitationsphysik (Albert-Einstein-Institut), \\ Callinstrasse 38, 30167 Hannover, Germany \\ (Received 2 August 2004; published 10 January 2005)
}

\begin{abstract}
We report on the demonstration of broadband squeezed laser beams that show a frequency-dependent orientation of the squeezing ellipse. Carrier frequency as well as quadrature angle were stably locked to a reference laser beam at $1064 \mathrm{~nm}$. This frequency-dependent squeezing was characterized in terms of noise power spectra and contour plots of Wigner functions. The latter were measured by quantum state tomography. Our tomograph allowed a stable lock to a local oscillator beam for arbitrary quadrature angles with $\pm 1^{\circ}$ precision. Frequency-dependent orientations of the squeezing ellipse are necessary for squeezed states of light to provide a broadband sensitivity improvement in third-generation gravitational-wave interferometers. We consider the application of our system to long-baseline interferometers such as a future squeezed-light upgraded GEO 600 detector.
\end{abstract}

DOI: 10.1103/PhysRevA.71.013806

PACS number(s): 42.50.Dv, 04.80.Nn, 42.65.Yj, 42.50.Lc

\section{INTRODUCTION}

Gravitational waves (GWs) were long ago predicted by Einstein using the theory of general relativity, but so far have not been directly observed [1]. Currently, an international array of first-generation, kilometer-scale laser interferometric gravitational-wave detectors, consisting of GEO $600[2,3]$, LIGO [3,4], TAMA 300 [5], and VIRGO [6], targeted at gravitational waves in the acoustic band from $10 \mathrm{~Hz}$ to $10 \mathrm{kHz}$, is going into operation. These first-generation detectors are all Michelson interferometers with suspended mirrors. Injecting a strong carrier light from the bright port, the antisymmetric mode of arm-length oscillations (e.g., excited by a gravitational wave) yields a sideband modulation field in the antisymmetric (optical) mode which is detected at the dark output port. To yield a high sensitivity to gravitational waves, these interferometers have long arm lengths of $300 \mathrm{~m}$ up to $4 \mathrm{~km}$ and high circulating laser powers, realized by additional arm cavities and power recycling [7]. Further improvement in sensitivity can be achieved by signal recycling [8], an advanced technique which will be implemented in second-generation GW interferometers and is already successfully implemented in GEO $600[9,10]$.

It was first proposed by Caves [11] that squeezed light injected into the dark port of a GW interferometer can reduce the high laser power requirements. In subsequent experiments shot-noise reduction from squeezed light was demonstrated in table-top interferometers [12-14]. Unruh [15] and others [16-19] have found and proven in different ways that quantum correlated light, e.g., squeezing at a quadrature angle of $45^{\circ}$, can reduce the quantum noise below the socalled standard quantum limit [20] at least at some frequencies. It was also shown that squeezed light with a frequencydependent orientation of the squeezing ellipse can reduce the quantum noise over the complete spectrum. In all cases interferometer topologies without signal recycling were considered. Recently Harms et al. [21] have generally shown that signal-recycled interferometers will benefit from squeezed light similarly to conventional interferometers as mentioned above. This result has strongly motivated further investigations on squeezed light because second-generation detectors like Advanced LIGO will combine arm cavities and signal recycling (the so-called resonant sideband extraction $[22,23])$ and will be quantum-noise limited over a substantial fraction of the detection spectrum. We emphasize that injected squeezed vacuum states can lead to a broadband sensitivity improvement, even at the two resonance frequencies of the detuned (signal tuned) signal recycling cavity, i.e., the optical resonance $[8,24,25]$ and the optomechanical (optical spring) resonance [26-28]. In [21] it was further shown that for a fixed readout homodyne angle two detuned filter cavities can provide the optimal frequency dependence for initially frequency-independent squeezed light. In [29] we investigated the high-frequency limit of signal-recycled gravitational-wave detectors. For detection frequencies above $\approx 1 \mathrm{KHz}$, GEO 600 is expected to be quantum-noise (shot-noise) limited; thus a reduction of quantum noise would lead to a sensitivity improvement without further reduction of other noise sources, such as thermal noise. In the shot-noise-limited regime of a signal-recycled interferometer a single filter cavity can provide the optimal frequency dependence for the squeezing ellipse. In that case the filter cavity should exactly match the interferometer's signalrecycling cavity and needs to be locked to the same signal sideband frequency but with the opposite sign. Figure 1 shows a proposal according to [29] of how such a filter cavity could be implemented using parts of the signal-recycling cavity and additional optics.

In this paper we present what we believe to be the first demonstration of a squeezed-source/single-filter cavity system, that provides a frequency-dependent squeezed laser beam. Long-term stable control has been achieved that enabled a detailed tomographic characterization of the nonclassical laser beam generated. Cooperation of two identical cavities with opposite detunings, so to speak a signalrecycling cavity and a filter cavity, has been inferred from our experimental data. 


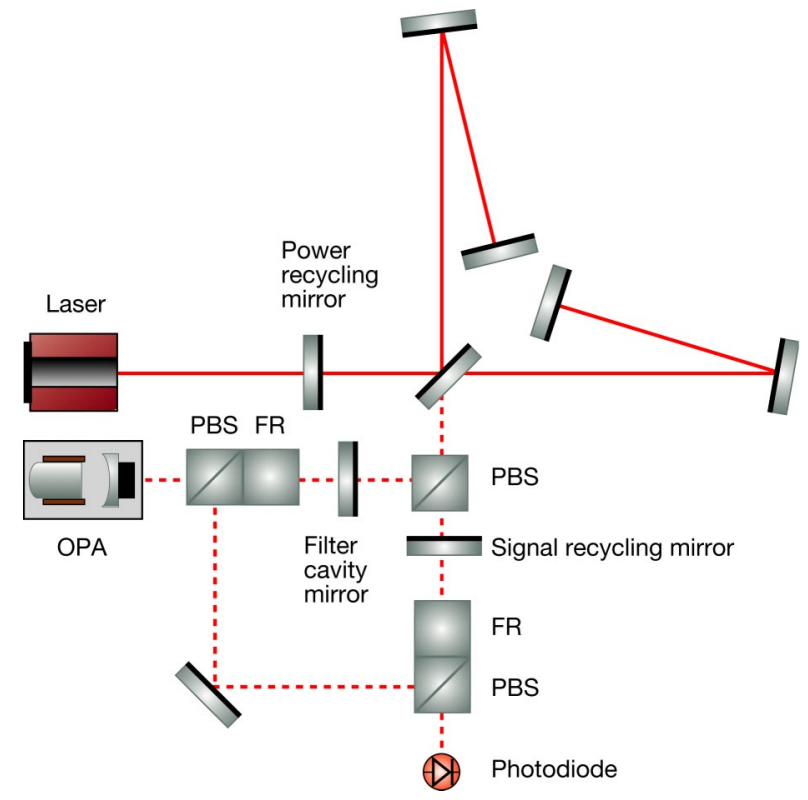

FIG. 1. (Color online) Interferometer topology of the GEO 600 gravitational-wave detector with additional filter cavity mirror, polarizing optics, and squeezed-light input from an optical parametric amplifier (OPA). Parts of the signal-recycling cavity are used to form a filter cavity that provides the desired frequency dependence of squeezed light applied to the dark port as proposed in [29]. For high frequencies (above $1 \mathrm{kHz}$ ) a single filter cavity is sufficient to gain a broadband sensitivity improvement from injected squeezed light. PBS, polarizing beam splitter; FR, Faraday rotator.

\section{SQUEEZED STATES OF LIGHT}

A single mode of the electromagnetic field can be described by its field amplitude or annihilation operator $\hat{a}(\omega)$, which has the commutation relation $\left[\hat{a}(\omega), \hat{a}^{\dagger}(\omega)\right]=1$. The non-Hermitian operator $\hat{a}(\omega)$ can be decomposed according to

$$
\begin{aligned}
& \sqrt{\frac{\omega_{0}+\Omega}{\omega_{0}}} \hat{a}\left(\omega_{0}+\Omega\right)=\frac{\hat{a}_{1}(\Omega)+i \hat{a}_{2}(\Omega)}{\sqrt{2}}, \\
& \sqrt{\frac{\omega_{0}-\Omega}{\omega_{0}}} \hat{a}^{\dagger}\left(\omega_{0}-\Omega\right)=\frac{\hat{a}_{1}(\Omega)-i \hat{a}_{2}(\Omega)}{\sqrt{2}},
\end{aligned}
$$

where $\hat{a}_{1,2}(\Omega)$ are the amplitude (subscript 1) and phase (subscript 2) quadrature operators of the two-photon formalism acting in the space of modulation frequencies $\Omega$ [30]. Correspondingly, the carrier frequency of the field is denoted by $\omega_{0}$. The discrete commutation relation $\left[\hat{a}_{1}(\Omega), \hat{a}_{2}^{\dagger}(\Omega)\right]=i$ follows directly from the commutation relation of $\hat{a}(\omega)$ and $\hat{a}^{\dagger}(\omega)$. This relation places a fundamental limitation on how well one quadrature of an optical beam can be known, given some knowledge of the orthogonal quadrature. This can be expressed by the uncertainty relation

$$
\Delta^{2} \hat{a}_{1}(\Omega) \Delta^{2} \hat{a}_{2}(\Omega) \geqslant \frac{1}{4}
$$

where the operator variances are denoted by

$$
\Delta^{2} \hat{a}_{1,2}=\frac{1}{2}\left\langle\hat{a}_{1,2} \hat{a}_{1,2}^{\dagger}+\hat{a}_{1,2}^{\dagger} \hat{a}_{1,2}\right\rangle-\left|\left\langle\hat{a}_{1,2}\right\rangle\right|^{2} .
$$

For pure states minimum uncertainty is given by the equal sign in Eq. (3). For conciseness where possible we omit the explicit frequency dependence $(\Omega)$ and since $\Omega \ll \omega_{0}$, we introduce the following approximation to Eq. (1):

$$
\begin{aligned}
& \hat{a}_{1}=\frac{a+a^{\dagger}}{\sqrt{2}}, \\
& \hat{a}_{2}=\frac{a-a^{\dagger}}{\sqrt{2 i}} .
\end{aligned}
$$

We need to keep in mind that amplitudes on the right-hand side act at different sideband frequencies; however, for all practical purposes, the quadrature amplitudes are Hermitian operators and therefore will be used as representatives of measurement results. With their continuous eigenvalue spectra these operators span the phase space where the Wigner function is defined. An arbitrary quadrature in this phase space can be defined as

$$
\hat{a}_{\theta}=\frac{1}{\sqrt{2}}\left(\hat{a} e^{-i \theta}+\hat{a}^{\dagger} e^{i \theta}\right)
$$

or

$$
\hat{a}_{\theta}=\hat{a}_{1} \cos \theta+\hat{a}_{2} \sin \theta .
$$

We move to the rotating frame at carrier frequency $\omega_{0}$ where the carrier (local oscillator) stands still on the real axis. Using a photodiode and demodulating the photoelectric current at $\Omega$ we actually observe the beat of the two sidebands contrarily rotating at $\pm \Omega$ with the carrier. If some apparatus changes the phase of only one sideband by the angle $\Phi$ keeping the carrier as well as the other sideband unchanged, we find that the quadrature angle of constructive interference changes by half the amount of the single sideband rotation,

$$
\begin{aligned}
\hat{a}_{\theta^{\prime}} & =\frac{1}{\sqrt{2}}\left(\hat{a} e^{i \theta}+\hat{a}^{\dagger} e^{-i(\theta+\Phi)}\right) \\
& =\frac{e^{-i \Phi / 2}}{\sqrt{2}}\left(\hat{a} e^{i \theta^{\prime}}+\hat{a}^{\dagger} e^{-i \theta^{\prime}}\right)
\end{aligned}
$$

$$
\text { with } \theta^{\prime}=\theta+\Phi / 2 \text {. }
$$

The transfer function $\rho$ of the reflection from a cavity with round-trip length $2 L$ and reflectivities $r_{1}$ and $r_{2}$ is given by

$$
\rho(\Omega)=\frac{r_{1}-r_{2} e^{2 i\left(\Omega-\Omega_{d}\right) L / c}}{1-r_{1} r_{2} e^{2 i\left(\Omega-\Omega_{d}\right) L / c}},
$$

where $\omega_{0}+\Omega_{d}$ is a resonance frequency. Hence sidebands are phase shifted by the amount of $\arg (\rho)$ which depends on the sideband frequency $\Omega$. As we will see in the next section, for our purpose we chose $r_{2}$ close to unity, resulting in a strongly overcoupled cavity, henceforth simply termed the filter cavity. 
Now we can consider squeezing at a certain frequency generated by the beat of two sidebands with the carrier. A squeezed state $\left|r_{s}, \theta, \alpha\right\rangle$ is obtained by applying the squeezing operator $S\left(r_{s}, \theta\right)=\exp \left\{-r_{s}\left[\exp (-2 i \theta) \hat{a}^{2}-\exp (2 i \theta) \hat{a}^{\dagger 2}\right]\right\}$ to a coherent state $|\alpha\rangle$ [31]. The resulting squeezed state comes with the desired noise power reduction of $\exp \left(-r_{s}\right)$ in quadrature $\hat{a}_{\theta}$ compared to coherent noise. Exactly as in classical sideband modulations the phase relation of the sidebands and the carrier determines the quadrature angle, here the angle of the squeezed quadrature.

In our experiment we used optical parametric amplification for squeezed-light generation. Initially the angle of the squeezed quadrature does not depend on frequency and the variance of the quadrature operator $\Delta^{2} \hat{a}_{\theta}$ reads

$$
\Delta^{2} \hat{a}_{\theta}=\cosh \left(2 r_{s}\right)-\sinh \left(2 r_{s}\right) \cos \left[2\left(\theta-\theta_{s}\right)\right] .
$$

The light beam noise powers are therefore white in frequency space at all angles when leaving the source. If then the squeezed beam is reflected from a detuned cavity having a small linewidth compared to its detuning the squeezing angle in phase space, $\theta_{s}$, will depend on the sideband frequency by virtue of $\arg (\rho)$ in Eq. (10), yielding

$$
\theta_{s}(\Omega)=\theta_{\mathrm{OPA}}+\frac{1}{2} \arg [\rho(\Omega)] .
$$

Here $\theta_{\mathrm{OPA}}$ is the initial, frequency-independent, but variable angle of the squeezed quadrature.

Finally we define the time-dependent normalized quadrature fields $\hat{q}_{1,2, \theta}(\Omega, t)$ whose variances are directly proportional to our measurement quantities,

$$
\hat{q}_{1,2, \theta}(\Omega, t)=\hat{a}_{1,2, \theta}(\Omega) e^{-i \Omega t}+\hat{a}_{1,2, \theta}^{\dagger}(\Omega) e^{i \Omega t} .
$$

Note that the right-hand side of Eq. (3) is equal to 1 if the quadrature fields $\hat{q}_{1,2}(\Omega, t)$ are considered instead of its amplitudes.

\section{EXPERIMENT}

In our experimental setup (Fig. 2) we used an optical parametric amplifier (OPA) to produce a dim laser beam at $1064 \mathrm{~nm}$ carrying squeezed states at sideband frequencies from 3 up to about $30 \mathrm{MHz}$ limited by the linewidth of the OPA cavity. The OPA was constructed from a type I phasematched $\mathrm{MgO}: \mathrm{LiNbO}_{3}$ crystal inside a hemilithic (halfmonolithic) resonator $[32,33]$. The resonator was formed by a high-reflective (HR) coated crystal surface of $10 \mathrm{~mm}$ radius of curvature on one side and an externally mounted cavity mirror of $25 \mathrm{~mm}$ radius of curvature and of reflectivity $r=\sqrt{0.95}$ on the other side. The intracavity crystal surface was antireflection coated (AR) for 1064 and $532 \mathrm{~nm}$. The $\mathrm{MgO}: \mathrm{LiNbO}_{3}$ crystal and the output coupler were separated by a $24 \mathrm{~mm}$ air gap creating a cavity mode for the resonant $1064 \mathrm{~nm}$ light with a $34 \mu \mathrm{m}$ waist at the center of the crystal. The OPA was seeded through the HR-coated surface with a coherent beam of $15 \mathrm{~mW}$ at the fundamental wavelength and pumped through the coupler with $300 \mathrm{~mW}$ of the second harmonic $(532 \mathrm{~nm})$ which results in a classical gain of 5 . The green pump light simply double-passed the nonlinear crystal

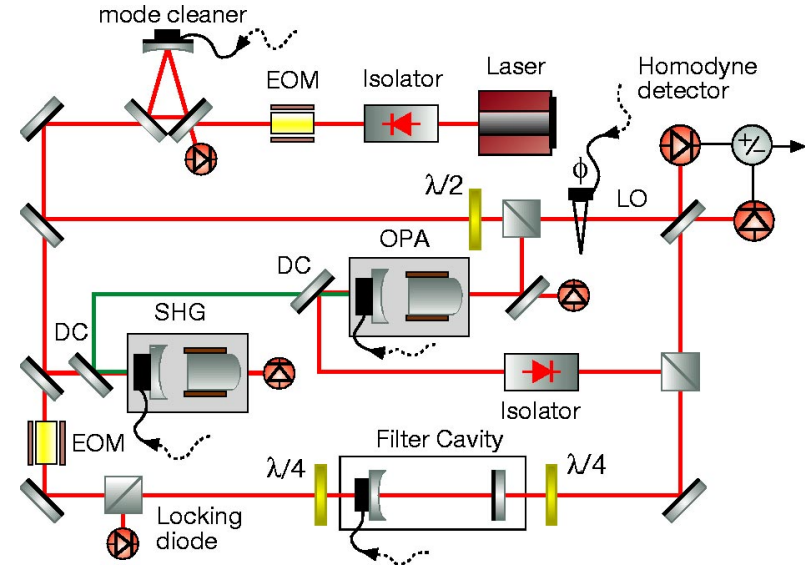

FIG. 2. (Color online) Schematic of the experiment. Amplitudesqueezed light is generated in an OPA cavity of controlled length. The detuned filter cavity provides frequency-dependent squeezing suitable for a broadband quantum-noise reduction of a shot-noiselimited signal-recycled interferometer. SHG, second-harmonic generation; EOM, electro-optical modulator; DC, dichroic mirror; LO, local oscillator; $\boldsymbol{\square}$, piezoelectric transducer.

because the OPA cavity was not resonant for the secondharmonic wavelength. The length of the OPA cavity was controlled using a sideband modulation technique based on an (intracavity) refractive index modulation on the $\mathrm{MgO}: \mathrm{LiNbO}_{3}$ crystal. This was achieved by a radiofrequency $(19.8 \mathrm{MHz})$ electric field applied to two copper plates that were placed on opposite sides of the nonlinear crystal. The error signal was determined by detecting the reflected seed beam and demodulating the resulting photocurrent at the used radio frequency. From the same beam we also generated an error signal for the phase difference of fundamental and second-harmonic waves inside the OPA. This enabled a stable lock to deamplification of the seed beam generating a dim amplitude-quadrature-squeezed beam of about $70 \mu \mathrm{W}$ at $1064 \mathrm{~nm}$. This control loop used a phase modulation on the second-harmonic pump at about 19.7 MHz that modulated the amplification of the OPA. A stable lock to amplification is similarly possible, thereby generating a phase-quadrature-squeezed beam. Note that both control locking loops did not require any measurement on the squeezed beam leaving the OPA.

The common laser source of all light beams was a monolithic nonplanar neodymium-doped yttrium aluminum garnet (Nd:YAG) ring laser of $2 \mathrm{~W}$ single-mode output power at $1064 \mathrm{~nm}$. Intensity noise below $2 \mathrm{MHz}$ was reduced by a servo loop acting on the pump diode current. To reduce excess noise above $2 \mathrm{MHz}$ the laser beam was transmitted through a mode cleaner ring cavity. The mode cleaner output was split into two beams as shown in Fig. 2; one of these beams was mode matched into a second-harmonic generator (SHG) to produce $532 \mathrm{~nm}$ light which in turn served as a pump field for our OPA. The SHG design [34] was similar to our OPA design and provided up to $600 \mathrm{~mW}$ of $532 \mathrm{~nm}$ light with up to $65 \%$ conversion efficiency. Parts of the remaining laser power were used as OPA seed radiation, as a local oscillator for homodyne detection $(18 \mathrm{~mW})$, and as a filtercavity length control beam $(10 \mathrm{~mW})$. 
The dim squeezed laser beam from the OPA was first passed through a Faraday isolator, preventing the OPA from any backscattered light. A $\lambda / 4$-wave plate turned the $s$-polarized beam into a circularly polarized beam which was then mode matched into our filter cavity (FC) and retroreflected into the homodyne detector for quantum state tomography. According to Fig. 1 we chose a linear filter-cavity design; the cavity was composed of a plane mirror of reflectivity

$r_{1}=\sqrt{0.97}$ and a concave mirror of reflectivity $r_{2}=\sqrt{0.9995}$; cavity length was $L=50 \mathrm{~cm}$ resulting in a linewidth of $1.47 \mathrm{MHz}$. The filter cavity was detuned by $\pm 15.15 \mathrm{MHz}$ throughout our measurements and stably locked to the lower or upper sideband, respectively. Length control was achieved by the Pound-Drever-Hall (PDH) locking technique utilizing a circularly polarized laser beam that carried $15 \mathrm{MHz}$ phase modulation sidebands and was coupled into the filter cavity from the opposite side.

All spectra presented in this paper were obtained from homodyne detector output photocurrents analyzed in a spectrum analyzer (Rohde\&Schwarz FSP3) with $100 \mathrm{kHz}$ resolution bandwidth and $100 \mathrm{~Hz}$ video bandwidth over the frequency range from 12 to $18 \mathrm{MHz}$. Each spectrum was at least $5 \mathrm{~dB}$ above the detection dark noise which was taken into account. The homodyne detector was constructed from two optically and electronically matched Perkin Elmer C30619 photodiodes. The phase difference of local oscillator and squeezed beam was controlled by using a combined dc-rf error signal. Usually a homodyne detector is controlled for an amplitude-quadrature measurement by using rf phase modulation sidebands on the dim (squeezed) signal beam or is controlled for a phase-quadrature measurement by utilizing the difference dc current from the two photodiodes as the error signal. In order to characterize quantum noise at all sorts of quadrature angles we required the optical phase at the homodyne beam splitter to be controllable at arbitrary phase angles. This was achieved by a sophisticated electronic control loop. As usual the actuator was a piezoelectric transducer shifting the optical path length of the local oscillator and the locking loop was closed by a PI element. The appropriate error signal $\left(S_{\text {err }}\right)$ was gained by a linear combination of the two linearly independent signals from above $\left[(\phi)_{\mathrm{rf}}\right.$ and $\left.(\phi)_{\mathrm{dc}}\right]$ which both sinusoidally depend on the phase angle $\phi$ :

$$
S_{\mathrm{err}}(\phi)=b(\phi)_{\mathrm{dc}}+(1-b)(\phi)_{\mathrm{rf}} .
$$

The parameter $b$ ranging from zero to one was computer controlled. The mapping of $\phi$ on $b$ is bijective in intervals of $[n \pi / 2,(n+1) \pi / 2)$. Those intervals could be appended by inverting the underlying signals to cover the whole range from 0 to $2 \pi$ thereby providing appropriate error signals for arbitrary quadrature angles.

In Figs. 3(a) and 4(a) we have plotted spectral noise powers of our frequency-dependent squeezed laser beam for various quadrature angles (see legends). Figure 3(a) shows spectra for a $+15.15 \mathrm{MHz}$ detuned filter cavity; Fig. 4(a) shows spectra when the cavity was detuned to $-15.15 \mathrm{MHz}$. The gray horizontal line represents the shot-noise limit in our measurement and was measured by blocking the squeezed
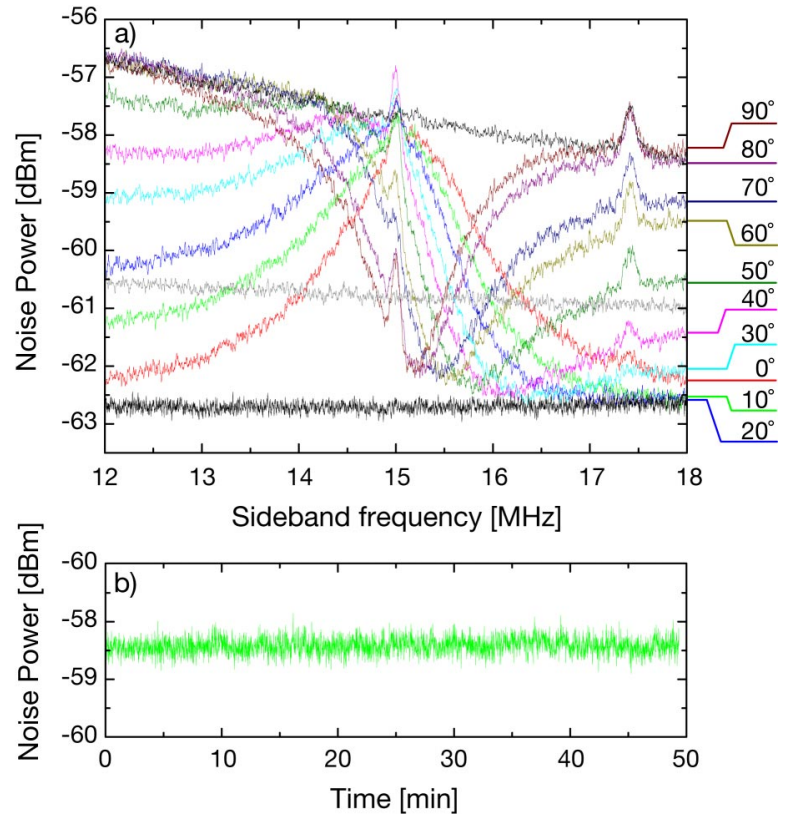

FIG. 3. (Color online) (a) Measured noise spectra of the frequency-dependent squeezed laser beam, stably locked to a homodyne oscillator at various quadrature angles. The measured quadratures show a smooth rotation from squeezed to antisqueezed quantum noise or vice versa. The filter cavity was detuned from the carrier frequency by $\Omega_{d}=15.15 \mathrm{MHz}$. (b) Demonstration of locking stability here for $10^{\circ}$ at $14.7 \mathrm{MHz}$.

beam. In both figures the array is bounded by measured traces of frequency-independent squeezing (lower black) or antisqueezing (top black). It can be seen that at high frequencies squeezing degraded due to the bandwidth of the OPA cavity. The frequency-independent squeezed and antisqueezed states were measured when a beam dump was placed inside the filter cavity. Thereby $3 \%$ loss on the squeezing due to the filter-cavity input coupler is introduced. The effect of another 3\% loss cannot be seen in the spectra. Note that all spectra in Figs. 3(a) and 4(a) were recorded by a conventional homodyne detector on a fully locked experiment. Figure 3(b) presents the locking stability. Here we locked the homodyne detector to measure the quadrature at about $10^{\circ}$ and recorded a zero span measurement at 14.7 $\mathrm{MHz}$ that last over $50 \mathrm{~min}$.

In an alternative experiment the frequency-dependent squeezed laser beam was detected by a homodyne quantum tomograph. Again the homodyne detector described above was used to turn the laser beam electric field properties at some quadrature angle into an electric current. Contrary to above, now the current was not fed into a spectrum analyzer but was mixed down with an electronic local oscillator at some radio frequency $\Omega$ and low-pass filtered. The final electric signal corresponds to a time series of measurement results on the chosen quadrature of the quantum state at sideband frequency $\Omega$. Quantum state tomography is a method to reconstruct an image starting from a set of its projections with rotating axis via a back transform algorithm. In our case the object to reconstruct is the Wigner function of the underlying quantum state and the projections are the probability 

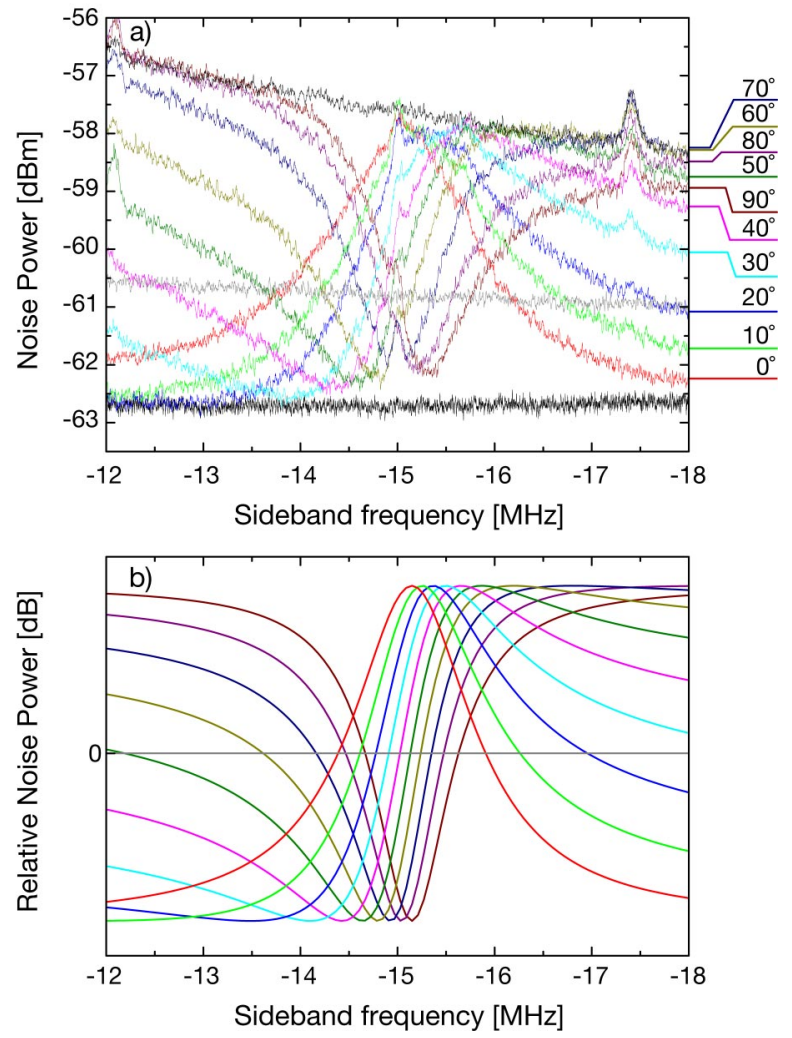

FIG. 4. (Color online) Measured noise spectra of the frequencydependent squeezed laser beam, stably locked to a homodyne oscillator at various quadrature angles. The measured quadratures show a smooth rotation from squeezed to antisqueezed quantum noise or vice versa. Here the filter cavity was detuned from the carrier frequency by $\Omega_{d}=-15.15 \mathrm{MHz}$. (b) Comparison with theoretical curves assuming pure states and perfect mode matching into the filter cavity.

densities at different quadrature angles. The transformation used is the inverse radon transform which is readily implemented in MATLAB. As described above our setup enabled a stably controlled measurement on arbitrary quadrature angles with a precision of $\pm 1^{\circ}$. This provides a solid basis for quantum state tomography. A LABVIEW program runs the following procedure for a predetermined set of angles: (1) Set the $b$ parameter [see Eq. (14)] for the next quadrature angle to be measured; (2) wait half a second for the control loop to stabilize; (3) acquire measurement values on the locked quadrature; (4) Build histogram from the data. The Wigner function was calculated afterward from the set of histograms. In Fig. 5 the result is shown for the quantum state at $14.1 \mathrm{MHz}$ sideband frequency (for discussion see next section). The resolution bandwidth was set to $100 \mathrm{kHz}$. For reconstruction altogether 100000 quadrature values were measured divided up on 100 equidistant quadrature angles. To characterize our frequency-dependent laser beam we measured 24 Wigner functions at various sideband frequencies. For these measurements the detuned filter cavity was locked to sideband frequencies of $\Omega_{d}= \pm 15.15 \mathrm{MHz}$. The results for the lower sideband $\left(\Omega_{d}=-15.15 \mathrm{MHz}\right)$ are shown in Fig. 6 .

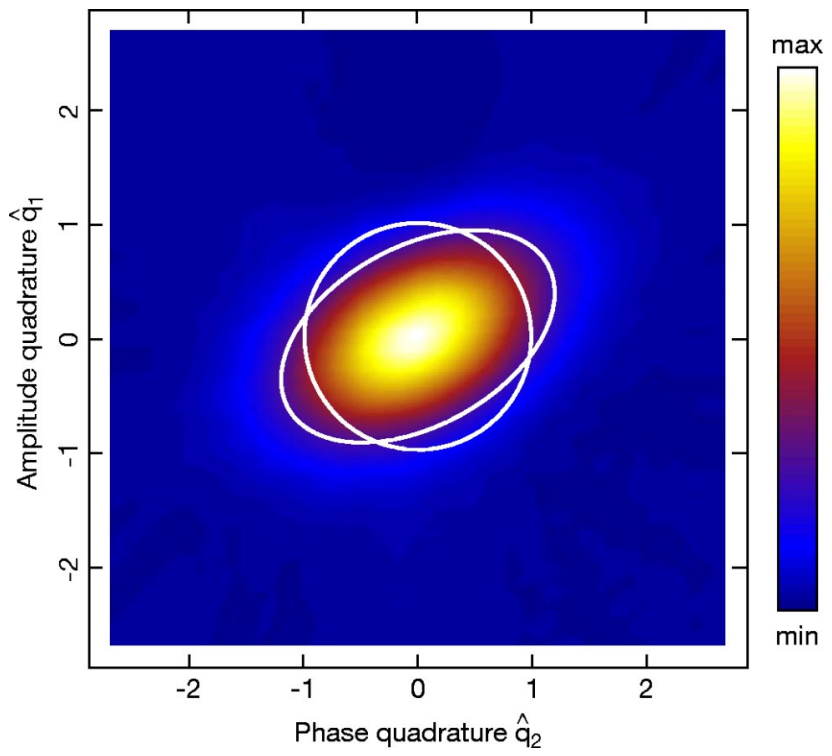

FIG. 5. (Color online) Measured contour plot of the Wigner function at $14.1 \mathrm{MHz}$ sideband frequency revealing the so-called squeezing ellipse. The white ellipse represents the standard deviation of the quantum noise; the white circle represents the size of the reference vacuum state. It is clearly visible that this state shows quantum correlations between phase and amplitude quadratures, i.e., squeezing at an angle of, here, about $40^{\circ}$.

\section{RESULTS AND DISCUSSION}

The colored spectra in Figs. 3(a) and 4(a) clearly show that the squeezed laser beam investigated carried frequencydependent squeezing. Homodyne detection of various quadrature angles revealed that squeezed quadratures appeared at different sideband frequencies in a spectrum of 12 to $18 \mathrm{MHz}$. For comparison the lowest and highest traces (in black) show common broadband squeezing and antisqueezing, respectively, where the squeezing ellipse does not rotate along the spectra of quantum states. In Figs. 4(a) and 4(b) we compare our results with theoretical curves assuming pure states, no optical losses, and perfect mode matching. Both pictures show a good qualitative agreement. Quantitative differences can be explained by overall losses on the squeezed beam, additional phase noise, and nonperfect mode matching of the squeezed beam into the filter cavity of $94 \%$. Especially those quadratures that show squeezing around the detuning frequency of $15.15 \mathrm{MHz}$ are strongly affected by nonperfect mode matching. The unmatched fraction is then antisqueezed and significantly deteriorates the achievable quantum-noise reduction. The phase modulation spikes at about 12.2 and $17.4 \mathrm{MHz}$ were stray fields from other experiments and picked up by the OPA. Due to thermal drifts in our polarizing optics the $15 \mathrm{MHz}$ modulation for filter cavity control also leaked into our homodyne detector. Although the signal could be completely canceled on short time scales by fine adjustment of the polarizing optics, the modulation frequency of $15 \mathrm{MHz}$ used for locking the filter cavity deteriorated our measurement, which lasted $30 \mathrm{~min}$ for a complete set of spectra. To keep the minima in Figs. 3(a) and 4(a) clearly visible and not hidden from the $15 \mathrm{MHz}$ modulation 

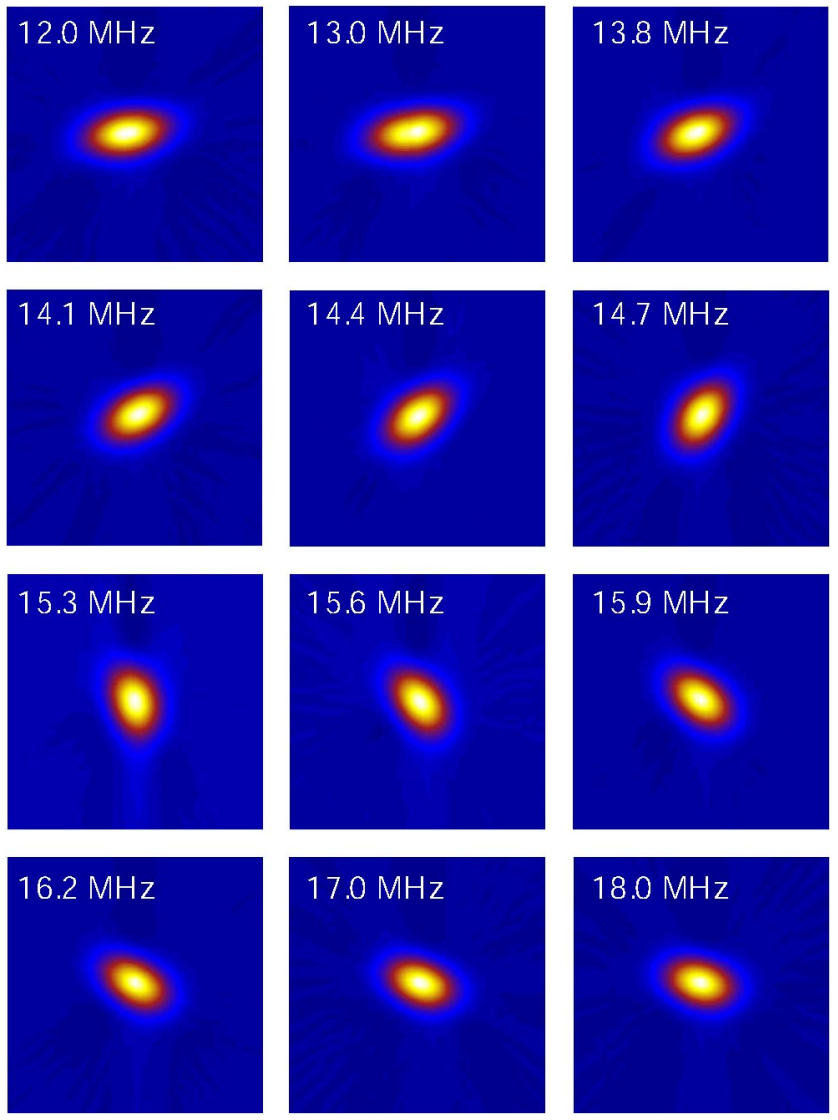

FIG. 6. (Color online) Contour plots of the squeezing ellipse at various sideband frequencies around $15 \mathrm{MHz}$, measured on the same continuous-wave laser beam. At $12 \mathrm{MHz}$ the squeezed quadrature was slightly off the vertical direction (amplitude quadrature); at higher frequencies the squeezing slowly rotates into the phase quadrature at $15 \mathrm{MHz}$ (not shown). For sideband frequencies far above $15 \mathrm{MHz}$ the squeezing will be measured again in amplitude quadrature. For each tomographic measurement the laser beam was phase locked to a reference beam and the quadrature angle stably controlled and stepwise rotated. The phase reference was given by a phase modulation at $19.8 \mathrm{MHz}$.

peak, we used an electric offset on the PDH error signal to lock the filter cavity at a sideband frequency of $15.15 \mathrm{MHz}$ instead of $15 \mathrm{MHz}$.

The overall squeezing in our measurement was detected to be $2 \mathrm{~dB}$ below shot noise. This value was primarily determined by optical losses. Compared to our previous squeezing results [35] in this experiment the losses were even higher due to the additional Faraday rotator and the filter cavity. Loss contributions from photodiode efficiencies, homodyne detector visibility, propagation losses, and OPA escape efficiency gave an overall loss on the squeezed states of $42 \%$.

An even clearer demonstration of frequency-dependent squeezing carried by our laser beam can be given in terms of Wigner function contour plots. In addition to the spectra discussed above, we did tomographic quantum state measurements [36,37] at frequencies between 12 and $18 \mathrm{MHz}$ as described in the previous section. Figure 5 presents a measured contour plot of the squeezing ellipse at a sideband frequency of 14.1 MHz. The white ellipse represents the

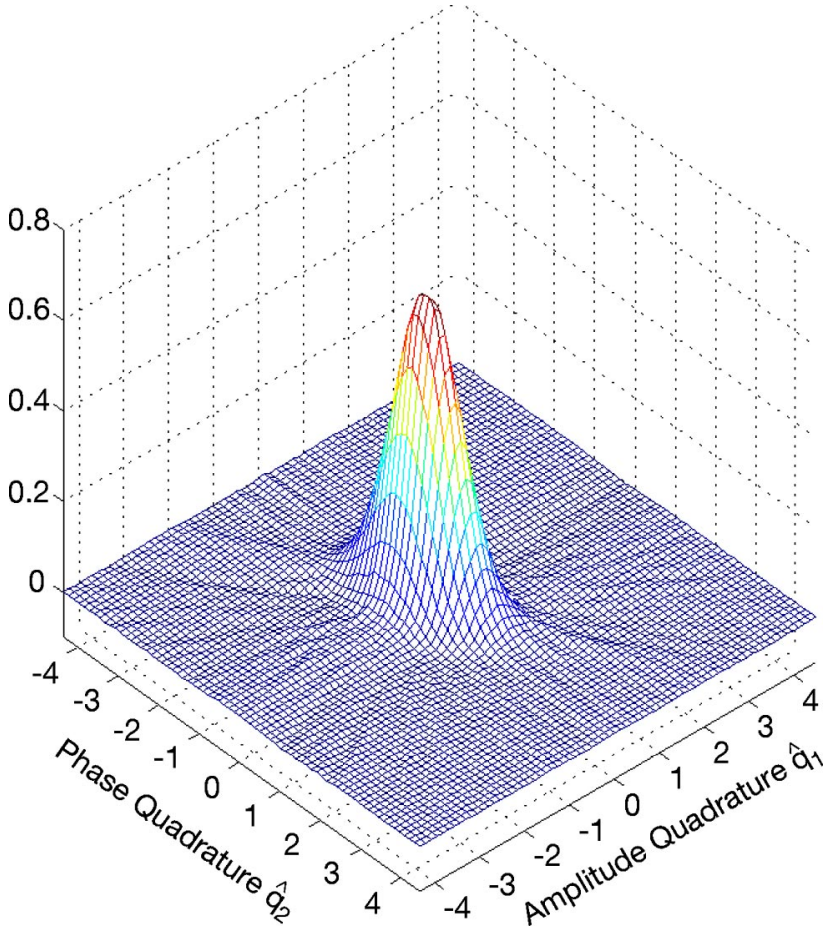

FIG. 7. (Color online) Example of three-dimensional Wigner function plot at $12.0 \mathrm{MHz}$ sideband frequency according to measurement data in Fig. 6. Comparing states at different frequencies, frequency-dependent squeezing is indicated by rotation along the vertical axis.

standard deviation of the quantum noise; the white circle represents the size of the reference vacuum state, normalized to unity. It is clearly visible that this state shows quantum correlations between phase and amplitude quadratures, i.e., squeezing at about $40^{\circ}$. Note that here the Wigner function is centered around the origin, saying that no coherent excitation of the modulation sideband was present. However, additional noise in the antisqueezed quadrature was clearly detected, since the area of the ellipse was determined to be 1.16 which can partly be attributed to optical losses on the squeezing and additional phase noise. Figure 6 shows our tomography results at various sideband frequencies using a detuned filter cavity with $\Omega_{d}=-15.15 \mathrm{MHz}$. For each tomographic measurement the homodyne detector was subsequently locked to 100 equidistant quadrature angles for a certain measurement time interval. An equivalent sequence was measured using a $+15.15 \mathrm{MHz}$ detuned filter cavity. The results are not explicitly shown but were used to plot curve (c) in Fig. 8 below. To the best of our knowledge we report on the first fully locked tomographic characterization of nonclassical states. In previous works the quadrature angles were slowly scanned, thereby averaging over a finite range of angles. In Fig. 7 an example of a three-dimensional Wigner function plot is given showing the state at $12.0 \mathrm{MHz}$ sideband frequency according to measurement data in Fig. 6. Figure 8 shows the effect of reflections from single detuned filter cavitities on the quadrature angle. Displayed are measured rotations of the squeezing ellipse deduced from our contour plots for $+15.15 \mathrm{MHz}$ (c) and $-15.15 \mathrm{MHz}$ (a) detuning. Altogether 24 measured Wigner function contour plots have been evalu- 


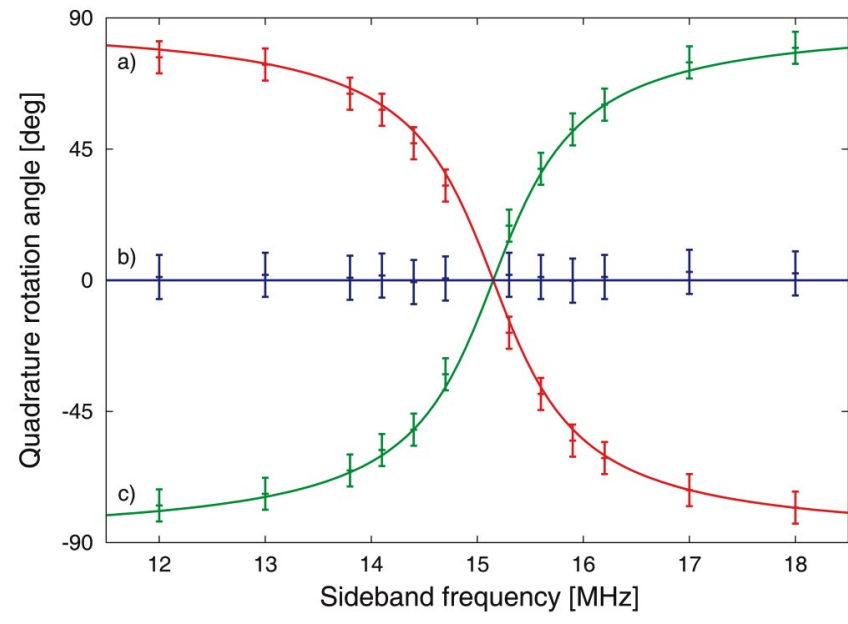

FIG. 8. (Color online) Measured rotations of squeezing ellipses. The two curves show the effects from $+15.15 \mathrm{MHz}$ (c) and from $-15.15 \mathrm{MHz}$ (a) detuned filter cavities. One of the cavities can be regarded as a signal recycling cavity of a shot-noise-limited interferometer. After two reflections the squeezing is again observed in the initial frequency-independent quadrature. The broadband squeezed quantum noise can then be adjusted to the signal carrying quadrature, giving a broadband sensitivity improvement for conventional homodyne readout.

ated. For every plot we determined the rotation angle of the squeezed quadrature with reference given by the initially squeezed amplitude quadrature. The two curved solid lines represent the theory according to Eq. (12). The very good agreement between experimental data and theory suggests that the approximation for small filter-cavity linewidth in Eq. (12) is valid for our experiment. From theory we expect that in any case the sum of both curved traces cancels to zero, shown as a horizontal line (b) in Fig. 8. This is indeed shown by our data points, thereby experimentally proving that two subsequent filter cavities that are symmetrically detuned by, in our case, $\pm 15.15 \mathrm{MHz}$ from the carrier lead to then an overall identical phase shift for carrier light and for all sideband frequencies. The effects of both cavities cancel each other.

Figure 8 is motivated by the fact that detuned cavities have become widely accepted in future gravitational-wave interferometers. As said in the Introduction the GEO 600 detector already uses a carrier-detuned (signal-tuned) socalled signal-recycling cavity. The second-generation detector Advanced LIGO will use a detuned signal extraction cavity. It is very likely that also third-generation detectors will make use of detuned cavities. Our research aimed for thirdgeneration GW interferometers where the ordinary coherent vacuum entering the interferometers dark port is replaced by a squeezed vacuum. Injected squeezed states will be reflected from the detuned cavity of the interferometer and thereby will gain a frequency-dependent phase shift. As a result the squeezed quadrature will not beat with the signal at all frequencies, resulting in a reduced detector bandwidth. Generically this can be circumvented by two or more appropriate filter cavities, which have been first proposed in [16] to enable sensitivities below the interferometer standard quantum limit. Contrary to [16] where ring cavities were proposed we used a linear filter cavity in correspondence to the design in Fig. 1, targeting the interferometer shot-noise limit. For frequencies above $1 \mathrm{kHz}$ the GEO 600 detector is not significantly affected by radiation pressure noise and a broadband sensitivity improvement from squeezed light can be achieved by a single detuned filter cavity. If the squeezed vacuum is reflected from a filter cavity with inverted detuning compared with the interferometer recycling cavity, both effects cancel. Then the squeezed states that are reflected from the interferometer show a frequency-independent orientation regarding the signal field and the shot-noise-limited sensitivity of the interferometer is improved over the complete spectrum according the degree of squeezing achieved.

\section{CONCLUSION}

Our results demonstrate frequency-dependent squeezing at sideband frequencies between 12 and $18 \mathrm{MHz}$. We have demonstrated a stably controlled system in a bench top experiment. Broadband but frequency-independent squeezing was provided by an optical parametric amplifier; frequencydependent orientation of the squeezing ellipse was achieved by reflecting the squeezing ellipse at a detuned filter cavity. For shot-noise-limited signal-recycled interferometers with homodyne readout at a fixed angle our scheme provides a broadband sensitivity improvement. Required cooperation of two identical cavities with opposite detunings, so to speak a signal-recycling cavity and a filter cavity, has been inferred from our experimental data. We used carrier light at $1064 \mathrm{~nm}$ which is the wavelength for current gravitational-wave interferometers; squeezing at acoustic frequencies was recently demonstrated for the same carrier wavelength [38]. Our scheme can be scaled to long baselines and acoustic frequencies using current technology.

\section{ACKNOWLEDGMENTS}

This work has been supported by the Deutsche Forschungsgemeinschaft and is part of Sonderforschungsbereich 407. We also acknowledge Jan Harms for his contributions to the theory section and Joshua R. Smith for his invaluable suggestions that contributed to the form and clarity of this paper. 
[1] K. S. Thorne, in 300 Years of Gravitation, edited by S. W. Hawking and W. Isreal (Cambridge University Press, Cambridge, England, 1987), pp. 330-458.

[2] B. Willke et al., Class. Quantum Grav. 19, 1377 (2002).

[3] B. Abbott et al., Nucl. Instrum. Methods Phys. Res. A 517, 154 (2004).

[4] A. Abramovici et al., Science 256, 325 (1992).

[5] M. Ando et al., Phys. Rev. Lett. 86, 3950 (2001).

[6] F. Acernese et al., Class. Quantum Grav. 21, S709 (2004).

[7] R. W. P. Drever et al., in Quantum Optics, Experimental Gravitation, and Measurement Theory, edited by P. Meystre and M. O. Scully (Plenum, New York, 1983), pp. 503-514.

[8] B. J. Meers, Phys. Rev. D 38, 2317 (1988).

[9] H. Grote et al., Class. Quantum Grav. 21, S473 (2004).

[10] J. R. Smith et al., Class. Quantum Grav. 21, S1737 (2004).

[11] C. M. Caves, Phys. Rev. D 23, 1693 (1981).

[12] M. Xiao, L.-A. Wu, and H. J. Kimble, Phys. Rev. Lett. 59, 278 (1987).

[13] P. Grangier, R. E. Slusher, B. Yurke, and A. LaPorta, Phys. Rev. Lett. 59, 2153 (1987).

[14] K. McKenzie et al., Phys. Rev. Lett. 88, 231102 (2002).

[15] W. G. Unruh, in Quantum Optics, Experimental Gravitation, and Measurement Theory (Ref. [7]), p. 647.

[16] H. J. Kimble, Y. Levin, A. B. Matsko, K. S. Thorne, and S. P. Vyatchanin, Phys. Rev. D 65, 022002 (2001).

[17] J. Gea-Banacloche and G. Leuchs, J. Mod. Opt. 34, 793 (1987).

[18] M. T. Jaekel and S. Reynaud, Europhys. Lett. 13, 301 (1990).

[19] A. F. Pace, M. J. Collett, and D. F. Walls, Phys. Rev. A 47, 3173 (1993).
[20] V. B. Braginsky and F. Y. Khalili, in Quantum Measurement, edited by K. S. Thorne (Cambridge University Press, Cambridge, Englnad, 1992).

[21] J. Harms et al., Phys. Rev. D 68, 042001 (2003).

[22] J. Mizuno et al., Phys. Lett. A 175, 273 (1993).

[23] G. Heinzel et al., Phys. Lett. A 217, 305 (1996).

[24] G. Heinzel et al., Phys. Rev. Lett. 81, 5493 (1998).

[25] A. Freise et al., Phys. Lett. A 277, 135 (2000).

[26] A. Buonanno and Y. Chen, Class. Quantum Grav. 18, L95 (2001).

[27] A. Buonanno and Y. Chen, Phys. Rev. D 64, 042006 (2001).

[28] A. Buonanno and Y. Chen, Phys. Rev. D 65, 042001 (2002).

[29] R. Schnabel, J. Harms, K. A. Strain, and K. Danzmann, Class. Quantum Grav. 21, S1045 (2004).

[30] C. M. Caves and B. L. Schumaker, Phys. Rev. A 31, 3068 (1985); B. L. Schumaker and C. M. Caves, ibid. 31, 3093 (1985).

[31] D. F. Walls and G. J. Milburn, Quantum Optics (Springer, Berlin, 1995).

[32] K. Schneider, M. Lang, J. Mlynek, and S. Schiller, Opt. Express 2, 59 (1998).

[33] W. P. Bowen, N. Treps, R. Schnabel, and P. K. Lam, Phys. Rev. Lett. 89, 253601 (2002).

[34] D. A. Shaddock et al., J. Opt. A, Pure Appl. Opt. 2, 400 (2000).

[35] R. Schnabel et al., Opt. Commun. (to be published).

[36] U. Leonhardt, Measuring the Quantum State of Light (Cambridge University Press, Cambridge, England, 1997).

[37] G. Breitenbach and S. Schiller, J. Mod. Opt. 44, 2207 (1997).

[38] K. McKenzie et al., e-print quant-ph/0405137. 